\title{
Blade Tip Timing Vibration Monitoring Method Based on Fiber Bragg Grating
}

\author{
Fei WU*, Lei LIANG, Junya XING, Lin WANG, and Lang JIA \\ School of Mechanical and Electronic Engineering, Wuhan University of Technology, Wuhan, 430070, China \\ *Corresponding author: Fei WU_ E-mail: wufei@whut.edu.cn
}

\begin{abstract}
The ventilators have been vividly called "the lungs of mine". The rotating blades are the core parts of a ventilator, they can influence the safety and reliability of the ventilator. This paper will use the tip-timing method based on the fiber Bragg grating magnetically coupling sensor to study and analyze the ventilator blade vibration, in order to realize long-distance and non-contact real-time online safety monitoring of blade vibration. Compared with the electronic sensorand fiber intensity reflective sensor, the fiber grating coupling magnetic sensor has such advantages as explosion-proof, working at harsh environment with humid air, dust and greasy dirt, capable of achieving long-distance signal transmission, and joining easily with other fiber Bragg grating sensors to form a network in order to achieve multi-parameter distributed online monitoring.
\end{abstract}

Keywords: Blade vibration, fiber Bragg grating, magnetic coupling, sensor

Citation: Fei WU, Lei LIANG, Junya XING, Lin WANG, and Lang JIA, "Blade Tip Timing Vibration Monitoring Method Based on Fiber Bragg Grating," Photonic Sensors, 2014, 4(2): 188-192.

\section{Introduction}

The large ventilator in the coal mine, which is vividly called "the lungs of mine", is one of the key equipments of mining and is an important part of the ventilation system. The mine ventilator plays a vital role in reducing the probability of explosions and increasing the safety of mine workers. In order to improve the mining safety and safety management level, the governments pay more attention to online monitoring of the ventilator, which improves the development of online monitoring of the mine safety [1]. In recent years, with the development of the optical fiber technology, this technology is quickly applied in various safety monitoring places because of its low cost, long-term stability, small size, low weight, anti-electromagnetic interference, environmental adaptability, easy to implement, and other advantages.

\section{Blade failures and their main forms of vibration}

Blade failures can be divided into two sorts by the extent: injury and damage. The blade injury is the state that the blade loses some performance but can still operate normally. Some injured blades can be put back into service after repair. Rusting, open welding, pitting, honeycombing, and getting scratched are the main blade injury forms. The blade damage is the state that the blade is seriously injured and cannot be put into the normal use. Twisting cracking, breaking, and secondary getting injured are the main forms of blade damages [2]. The cause of blade failures is manifold. Looking from the life cycle of the blades, the first reason is the designing 
and manufacturing problem. The defects of the material itself, the improper designing, and the unqualified manufacturing precision may all lead to the blade failures.

Secondly, withstanding excessive stresses will also result in blade failures, which are mainly caused by vibration. The working blades have to bear the rotating centrifugal force, the alternating stress caused by blade vibration, and the wind pressure in the protective cover. When the cumulative operating time reaches to a certain point, the blades will have fatigue cracks. If blades continue to run, the size of cracks will stretch, when the cracks reach to a certain degree, the blades will break. The flying debris of the high speed rotating broken blade may injure or damage other blades. The end result is that all the blades in the same blade-group are broken.

According to the vibration direction, the blade vibration can be divided into bending vibration, twisting vibration, and composite vibration.

\section{Working principle of vibration monitoring with the tip-timing method}

The working principle of the vibration monitoring with the tip-timing method is to measure the time-sequence signals generated from each tip-timing sensor, which are associated with rotating blades [3].

In each cycle of blade's normal rotation without vibration, the time that a blade runs through the sensors depends on the rotation speed of the blade and the mounting location of the sensors; when vibration occurs in the blade, the time of the blade through tip-timing sensors will change, either earlier or later, and there will be a time difference $\Delta t$ between the normal and vibrating rotations. At this time, the time that the blade running through the sensors depends not only on the blade rotation speed, but also on the frequency and amplitude of blade vibration. With the reference data provided by the relevant sensors and the measured data of time difference sequence $\{\Delta t\}$, we can know the amplitude and frequency of the blade vibration, as shown in Fig. 1.

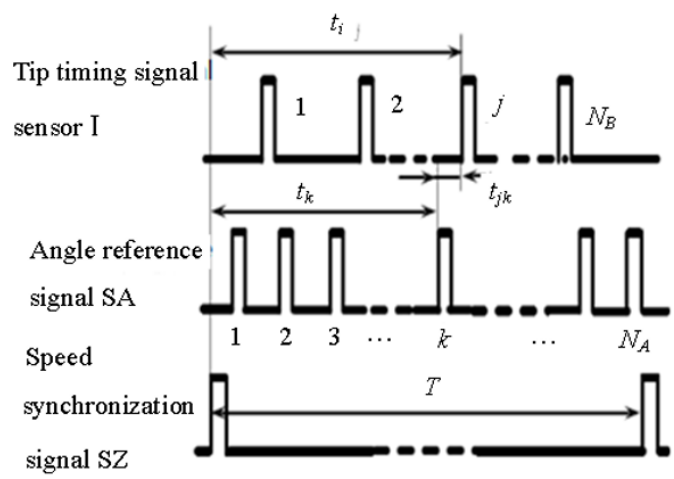

Fig. 1 Tip-timing method.

The vibration monitoring system with tip-timing method is shown in Fig. 2. Generally, it is composed of 5 parts, namely the synchronous speed sensor, the tip-timing sensor, the blade root sensor (which in most cases can be omitted), the data acquisition, the data processing and displaying.

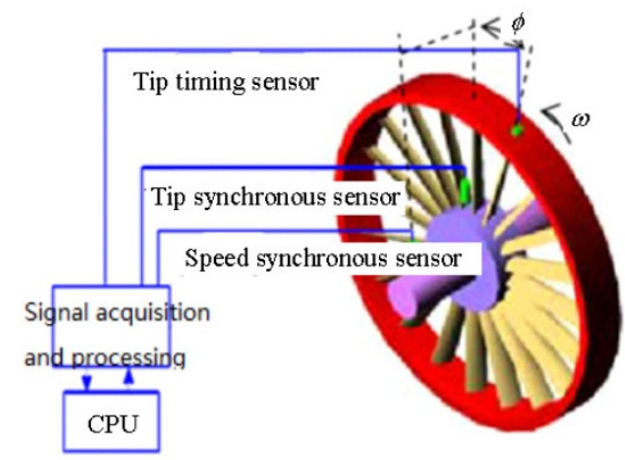

Fig. 2 Tip-timing measurement system.

Among the 5 parts, the mostly researched one is the tip-timing sensor, and the different principle of the tip-timing sensor corresponds to the different data collection method.

The tip-timing vibration system generally includes four main functions. The first function is to get blade speed through the synchronous speed sensor, then combining with the known fixed parameters of the blade, we can deduce the theoretical time sequence that the blade passes the tip timing sensor. The second is to obtain the actual time sequence that the vibrating blade passes a tip-timing sensor; The third is to obtain the vibrating 
speed of the blades, the time interval between the different time sequences and other parameters from the blade's rotating speed, the fixed known parameters, the theoretical and the real time sequence. The fourth is to analyze and process the data obtained and to obtain parameters of blade vibration, generally the amplitude, and then the frequency of the blade vibration can be obtained by the fast Fourier transform (FFT).

\section{Signal analysis of tip-timing measurement}

The blade vibration can be divided into synchronous and asynchronous vibrations. By test, we can obtain the tip-timing time sequence and the synchronous speed time sequence, but there are differences between these two time sequences in the amplitude frequency and phase of the extraction.

(1) Asynchronous vibration is that the blade vibration frequency is not an integer multiple of the frequency of the blade speed when the vibration occurs.

Whether or not there is an asynchronous blade vibration can be judged by the obtained time signal, the way of judgment is analyzed as following: firstly, to number all the blades according to a certain sequence; secondly, to extract the time sequence $\left\{t_{i}, j\right\}$ corresponding to the maximum signals which are generated by each blade when they pass the same tip-timing sensor, where $t_{i}$ denotes the time signal which is generated by the $i$-th blade when it pass the tip-timing sensor the $j$-th time, where $i$ and $j$ are the integers greater than zero. If two time difference sequences of two adjacent blade tips passing through the same tip-timing sensors $\left\{t \Delta i, j=t_{i}+1, j-t_{i}, j\right\}$ are not equal, there is an asynchronous vibration vane; if the time difference sequences $\{t \Delta i, j\}$ are equal, there is no asynchronous vibration vane.

Compared with the synchronous vibration, the monitoring of asynchronous vibration is relatively simple. Only two tip-timing sensors which are installed on the shell of the rotating machine can measure the amplitude sequences of all blades.

As shown in Fig. 3, the rotational speed of the impeller blades is $n$, when the impeller blade turns each round, the theoretical computation time and the actual time when a blade reaches the tip-timing sensor $\mathrm{B}$ are $t_{0}$ and $t_{i}$, respectively. $\tau_{i}$ is the interval of blade passing though two tip-timing sensors A and B. $T$ is the period of revolution of the impeller. After the $N$-round rotation of impeller blades, the sequence of $\left\{x_{i}\right\}$ of the blades vibration displacement can be obtained. When $x_{i}$ is set to a transient vibration displacement of the blade, we have the following equations:

$$
\begin{gathered}
x_{i}=v_{i}\left(t_{i}-t_{0}\right) \quad(i=1,2,3,4,5, \ldots, N) \\
v_{i} \approx(R \theta) / \tau_{i}-n .
\end{gathered}
$$

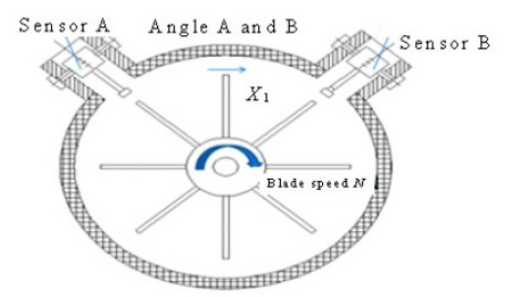

Fig. 3 Schematic diagram of blade vibration measurement.

In the above two equations, $v_{i}$ is the average vibration velocity of the blade tip; $R$ is the radius of the blade tip; $\theta$ is the mounting angle between the sensors A and B. As shown in Fig. 4, $x_{1}(n)$ and $x_{2}(n)$ present the sampling displacements of the rotating blade passing by Sensor A and Sensor B, respectively.

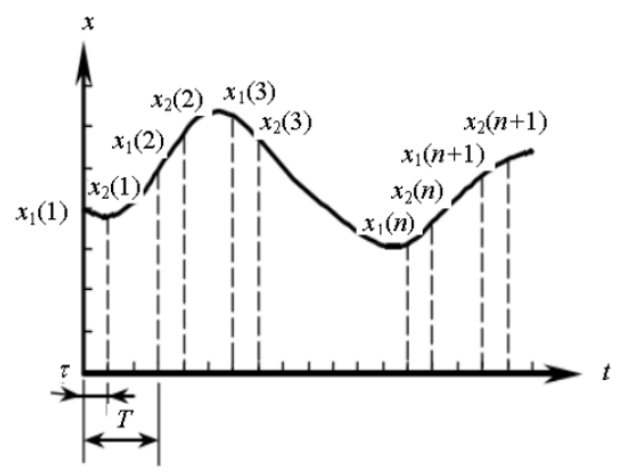

Fig. 4 Displacement sequence of blade asynchronous vibration. 
When the revolution number $N$ is large and the time duration is long enough, the maximum displacement $|x|_{\max }$ can be sampled by the blade tip vibration timing sensor. To eliminate the blade vibration displacement offset errors, the following formula is used to calculate the blade amplitude $A$ :

$$
A=\frac{\left(x_{\max }-x_{\min }\right)}{2}
$$

where $x_{\max }$ and $x_{\min }$ are the maximum and minimum values of the blade vibration displacement, respectively.

During the rotation of the blades, the rotational frequency of a blade is also the sampling frequency of the tip-timing sensor, which is far below the blade vibration frequency. Since this measurement does not meet the sampling theorem, the sequence of the blade vibration displacement transform FFT $\left\{x_{i}\right\}$ is not obtained by the actual vibration frequency of the blade, but leaving the actual frequency difference frequency portion; the integer frequency of the blade vibration can be obtained by the calculated dynamic frequency $m f_{r}$ of the blades. So the actual vibration frequency of the blade is

$$
f=(m \pm \Delta m) f_{r}
$$

where $f$ is the actual vibration frequency of the blade; $f_{r}$ is the frequency of the rotor speed; $m$ is the order of excitation which can be preferably chosen as an integer of $0,1, \cdots ; \Delta m$ is a fraction of less than 0.5 .

(2) Synchronous blade vibration has the characteristic that the blade vibration frequency is an integer multiple of the frequency of the blade rotating speed. When the impeller speed is constant, the sampling frequency of the tip-timing sensor also keeps constant, regardless of how many rounds of blade rotations, because the tip-timing sensors will collect the same blade vibration data of certain blade [4].

There are two ways in synchronization blade vibration measurement. The first is the indirect method, also known as the velocity vector side trace method, which is achieved by changing the rotational speed of the impeller vanes, thus changing the sampling frequency; when the rotating speed falls into the resonance area of the blade, the resonance amplitude and natural frequency can be measured The velocity vector side track method is currently one of the widely used methods, which needs only one or two sensors installed on the shell of the rotating machine to measure the blade vibration parameters. However, this method requires a constantly changing speed, so the device cannot be used in the on-line real-time monitoring, and the device cannot be used for monitoring constant speed conditions.

The second method is known as the maximum amplitude probability method, in which $5+2$ sensors are mounted on the shell of the rotating machine at a certain angle to achieve the better measurement of the blade vibration parameters. The mounting positions of the sensors are shown in Fig. 5. If only 3, 4 , or 5 sensors are installed, the orders with integer multiple of sensor numbers will not be properly measured.

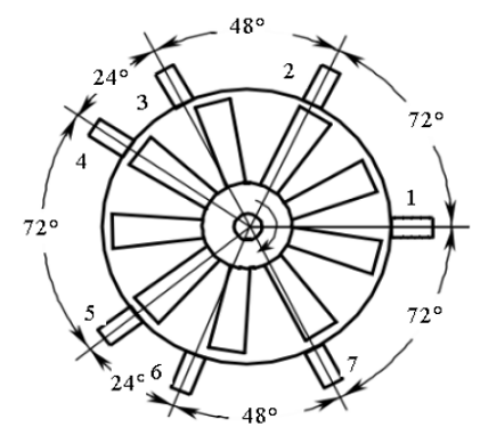

Fig. 5 Sensors arrangement.

The blade vibration displacement can be given by

$$
x_{i j}=A \sin \left(m \omega t_{i j}+\varphi\right)+C
$$

where $x_{i j}$ is the displacement measured when the blade passes the $j$-th sensor within the $i$-th rotation; $A$ represents the amplitude of the blade; $\omega$ represents the impeller blade speed; $t_{i j}$ is the time of the blade reaching the $j$-th sensor in the $i$-th lap; the angle $\varphi$ represents the initial phase angle.

$$
\because t_{i j}=\frac{\theta_{j}+i 2 \pi}{\omega}
$$




$$
\begin{gathered}
\omega t_{i j}=\theta_{j}+i 2 \pi \\
\therefore x_{i j}=A \sin \left(m i 2 \pi+m \theta_{j}+\varphi\right)+C .
\end{gathered}
$$

In (8), $\theta_{j}$ is the mounting angle of the $j$-th tip timing sensor. When the vibration occurs in the blades rotating, as long as the measured time reaches a certain value, the sensor can always capture the maximum time difference $\Delta \tau_{\max }$. The blade amplitude $A$ is proportional to the time difference $\Delta \tau$ $=\left|t_{i}-t_{0}\right|$. The maximum amplitude of the tip can be derived as long as the maximum time difference can be collected.

In the blade vibration, the probability to measure the maximum vibration amplitude is the same as that of amplitude distribution probability of the sine function, because the time difference $\Delta \tau$ is related to the blade amplitude $A$ and the phase of $\sin \left(m i 2 \pi+m \theta_{j}+\varphi\right)$.

When $\left|\sin \left(m i 2 \pi+m \theta_{j}+\varphi\right)\right|=1$, the blade amplitude $A$ can be calculated.

$$
\begin{gathered}
\because\left|\sin \left(m i 2 \pi+m \theta_{j}+\varphi\right)\right| \approx 1 \\
\therefore x_{i \max } \approx A+C .
\end{gathered}
$$

In (10),

$$
C=\sum_{i=1}^{N} x_{i j} / N
$$

where $N$ is the revolution number.

$$
\therefore A \approx x_{i \max }-C \text {. }
$$

From (8),

$$
\begin{gathered}
\sin \left(m i 2 \pi+m \theta_{j}+\varphi\right)=\frac{x_{i j}-C}{A} \\
m i 2 \pi+m \theta_{j}+\varphi=\arcsin \frac{x_{i j}-C}{A} .
\end{gathered}
$$

Then, we get

$$
m i 2 \pi+\mathrm{m} \theta_{j+1}+\varphi=\arcsin \left[\left(x i_{(j+1)}-C\right) / A\right] .
$$

From (13) and (14),

$$
m=\left(\arcsin \frac{x_{i(j+1)}-C}{A}-\arcsin \frac{x_{i j}-C}{A}\right) /\left(\theta_{j+1}-\theta_{j}\right) \text {. }
$$

The blade vibration frequency is obtained from (4):

$$
f=m f_{r} .
$$

\section{Conclusions}

The blade vibrations can be measured and monitored through the changes in the grating wavelengths that are crushed by the diaphragm vibration, which is generated by the permanent magnetic probe when a blade approaches these magnetic probe. During each blade rotating cycle, the fiber Bragg grating (FBG) demodulator demodulates the time signal corresponding to the central maximum wavelength, and this time signal is the required time pulse signal when the tip of the blade reaches the timing sensor. After the processing of time pulse sequence, the blade vibration parameters can be obtained.

The proposed blade vibration monitoring methods based on magnetic coupling FBG sensors have been proved to be feasible by theoretical and experimental verification, and the above two methods will be seriously considered in the further in-depth research.

Open Access This article is distributed under the terms of the Creative Commons Attribution License which permits any use, distribution, and reproduction in any medium, provided the original author(s) and source are credited.

\section{References}

[1] X. Lu, P. Yu, T. Yu, and C. Qiu, "PLC-based mine main man network monitoring," Coal Science and Technology, 2009, 37(1): 22-24.

[2] T. OuYang, "Rotating blade vibration performance testing technology research," Ph.D. dissertation, Tianjin University, 2008.

[3] M. L. Adams, Rotating machinery vibration: from analysis to trouble shooting. New York: Marcel Dekker, 2001.

[4] H. Y. Wang, "High-speed rotating blade vibration blade tip timing measurement methods and systems research," Ph.D. dissertation, Tianjin University, 2003. 